\title{
Factors associated with early cardiac complications following transcatheter aortic valve implantation with transapical approach
}

This article was published in the following Dove Press journal:

Pragmatic and Observational Research

\author{
Vasileios Patris' \\ Konstantinos \\ Giakoumidakis' \\ Mihalis Argiriou' \\ Katerina K Naka \\ Efstratios Apostolakis ${ }^{3}$ \\ Mark Field ${ }^{4}$ \\ Manoj Kuduvalli ${ }^{4}$ \\ Aung $\mathrm{Oo}^{4}$ \\ Stavros Siminelakis ${ }^{3}$ \\ 'Department of Cardiac Surgery, \\ "Evangelismos" General Hospital \\ of Athens, Athens, Greece; \\ ${ }^{2}$ Department of Cardiology, Medical \\ School, University of Ioannina, \\ loannina, Greece; ${ }^{3}$ Department of \\ Cardiothoracic Surgery, University \\ of loannina, loannina, Greece; \\ ${ }^{4}$ Department of Cardiothoracic \\ Surgery, Heart and Chest Hospital of \\ Liverpool, Liverpool, UK
}

Correspondence: Konstantinos

Giakoumidakis

Department of Cardiac Surgery,

"Evangelismos" General Hospital of

Athens, 45-47 Ipsilantou Street, Athens

10676, Greece

Tel +30 6973793489

$\mathrm{Fax}+302132041987$

Email kongiakoumidakis@gmail.com
Purpose: To estimate the incidence of postprocedural early cardiac complications among patients undergoing transcatheter aortic valve implantation, through transapical approach (TATAVI), and to identify factors independently associated with the occurrence of them.

Patients and methods: A retrospective cohort study of 90 patients, who had undergone TATAVI in a tertiary hospital of Liverpool, UK, during a 5-year period (September 2008-October 2013), was conducted. Data on patient demographics, periprocedural characteristics and cardiac complications presented within 30-day post TA-TAVI were collected, retrospectively, using the hospital's electronic database.

Results: The overall 30-day incidence of cardiac complications was estimated at $18.9 \%$ $(n=17 / 90)$. The rate of new onset of atrial fibrillation (AF), atrioventricular block requiring permanent pacemaker implantation, shockable cardiac arrest rhythm and cardiac tamponade was $11.1 \%, 3.3 \%, 2.2 \%$ and $2.2 \%$, respectively. Bivariate analysis found that absence of preoperative $\mathrm{AF}(p=0.01)$, receiving of oral inotropes preprocedurally $(p=0.01)$, intravenous inotropic support postprocedurally $(p=0.01)$ and requirement for postprocedural tracheal intubation $(p=0.001)$ were the main factors associated with increased probability for patient cardiac morbidity.

Conclusion: It seems that patients with absence of AF and oral inotropic support preprocedurally and those with post TA-TAVI mechanical ventilatory and intravenous inotropic support have greater probability to develop cardiac complications. This knowledge allows the early identification of high-risk patients and supports clinicians to apply both preventive and therapeutic interventions for the optimum patient management and care. In addition, administrators could allocate the health care system resources effectively.

Keywords: aortic valve stenosis, complications, retrospective studies, transcatheter aortic valve replacement

\section{Introduction}

Transcatheter aortic valve implantation (TAVI) is a well-documented, through highquality randomized controlled trials, therapeutic option for patients with symptomatic aortic stenosis, ${ }^{1}$ consisting not only the treatment of choice for inoperable patients ${ }^{2}$ but also a feasible alternative to surgery in patients at high ${ }^{1}$ and intermediate surgical risk..$^{3,4}$

It is estimated that during the 3-year period (2011-2014), more than 26,000 TAVI procedures had been performed at 348 centers in the USA with satisfactory patient outcomes. ${ }^{5}$ However, despite the high safety and effectiveness of TAVI procedure in appropriately selected patients groups, ${ }^{6}$ this therapeutic option has several periprocedural complications, including stroke, bleeding, vascular complications, acute kidney injury, requirement for permanent pacemaker (PPM) implantation, myocardial 
infarction, valve malposition, paravalvular leak and heart block. ${ }^{7,8}$ In the currently available published research, several patient and technical-related factors have been associated with postprocedural complications such as advanced age, female gender, diabetes mellitus, poor left ventricular ejection fraction, peripheral arterial disease, access route, size of the delivery system, history of stroke, prior and/or a new-onset atrial fibrillation (NOAF), baseline severe aortic regurgitation, severity of calcifications on the valve and the aortic arch. ${ }^{7,9}$

The literature review reveals that TAVI complications have mainly evaluated as risk factors for patient mortality in several patient series, ${ }^{10-13}$ and few are known to regard the periprocedural factors independently associated with TAVI complications, which could allow the early identification of patients at high risk for morbidity after TAVI. Moreover, this literature deficit is more apparent among those receiving TAVI through the transapical access, a patient subgroup with poorer outcome, compared with the most widely used and first choice approach, the transfemoral one. ${ }^{6,9,14}$ The purpose of the present study was to investigate the periprocedural parameters associated with early cardiac complications in patients after TAVI procedure through the transapical approach. Our study intents to contribute new data to TAVI complications prognosis limited body of knowledge in this specific transapically treated patients' subgroup.

\section{Patients and methods}

\section{Study design and participants}

We carried out a retrospective cohort study of 90 consecutive patients with severe aortic valve disease, who were admitted to a cardiothoracic surgery center of Liverpool, UK, for TAVI procedure, through a transapical approach, under general anesthesia, during a 5-year period (September 2008-October 2013). All patients were implanted with the Edwards SAPIEN-XT valve (Edwards Lifesciences Inc., Irvine, CA, USA). On the basis of our a-priori set study inclusion criteria, our sample included only patients with successful valve implantation, without requirement for conversion of TAVI procedure to an open surgical aortic valve replacement. Conversion to surgery was necessary in 1 patient, due to pericardial tamponade.

\section{Data collection}

Data collection was performed in November 2014, using the hospital's structured database (e-cardiac), which included demographic and periprocedural patient data. Specifically, patients' age, gender, weight, height, logistic EuroSCORE, comorbidity (diabetes mellitus, chronic renal insufficiency and hypercholesterolemia), cardiac patient history ( $Q$ wave myocardial infarction, previous cardiac surgery, preoperative coronary angiography, preoperative cardiac rhythm, oral inotropes and left ventricular ejection fraction), smoking status, preoperative serum creatinine $(\mathrm{Cr})$ levels, duration of both the postprocedural tracheal intubation and the postprocedural intravenous inotropic support, postprocedural volume of blood loss and postprocedural cardiovascular complications occurred within 30 days post valve implantation.

We recorded the 30-day cardiac morbidity post TAVI, considering the following end points as cardiac complications: new atrioventricular block requiring PPM implantation, NOAF, cardiac arrest rhythms, including pulseless ventricular tachycardia (VT) and/or ventricular fibrillation (VF) and cardiac tamponade diagnosed echocardiography when patient condition allows or by emergency mediastinal re-exploration when patient hemodynamics were very tenuous. We considered NOAF as the occurrence of AF in patients who had sinus rhythm preprocedurally. Finally, we defined as postprocedural tracheal intubation the invasive patient ventilatory support through an endotracheal tube after TAVI procedure completion.

\section{Statistical analysis}

We presented continuous variables as median and interquartile range, while categorical variables are depicted as numbers and percentages. Continuous variables were converted into binary variables using their median values as the criterion for separation (cut-off point). However, for body mass index and serum $\mathrm{Cr}$ levels the relevant cut-off points were the values of 24.9 $\mathrm{kg} / \mathrm{m}^{2}$ and $1.4 \mathrm{mg} / \mathrm{dL}$, respectively. ${ }^{15}$ Chi square test, Fisher's exact test, chi square trend test and student's $t$-test were performed for bivariate associations and $p$-values $<0.01$ were defined as significant, aiming to reduce error type I. The IBM SPSS 21.0 for Windows software (IBM Corporation, Armonk, NY, USA) was used for our statistical analysis purposes.

\section{Ethics}

Taking into account our study design and methods (retrospective observational study, with no randomization and therapeutic interventions), there was no need for ethical approval by the ethics committee of the "Heart and Chest Hospital" of Liverpool, UK, according to the hospital practice. In addition, to review their medical records, patient consent was not required by the ethics committee, based on the hospital practice. Precautions were taken to ensure the confidentiality of patient data during the data collection process. Research 
was conducted in conformity with the ethical standards of both the responsible institutional committee for human experimentation and the Declaration of Helsinki.

\section{Results}

The median (interquartile range [IQR]) age of our cohort was 83 (10) years and we had an equal number of males and females in our sample. The median (IQR) logistic EuroSCORE was $20(18.5) \%$. The main demographic, clinical and periprocedural patient characteristics are summarized in Table 1. In addition, as shown in Table 2, the overall incidence of cardiac postprocedural complications was estimated at $18.9 \%(\mathrm{n}=17 / 90)$. Specifically, 3 patients $(3.3 \%)$ developed atrioventricular block requiring PPM implantation, 10 of them (11.1\%) had an NOAF, 2 patients (2.2\%) presented a

Table I Demographic, clinical and periprocedural patient characteristics

\begin{tabular}{|c|c|}
\hline Variables & Median ( $\pm I Q R)$ \\
\hline Age (years) & $83(10)$ \\
\hline Logistic EuroSCORE (\%) & $20(18.5)$ \\
\hline Postprocedural intubation time (hours) & $0(3)$ \\
\hline Creatinine levels (mg/dL) & I.I (0.6) \\
\hline Postprocedural blood loss volume (mL) & $23(200)$ \\
\hline \multicolumn{2}{|l|}{ Gender, n (\%) } \\
\hline Male & $45(50.0)$ \\
\hline Female & $45(50.0)$ \\
\hline Diabetes, n (\%) & $20(22.2)$ \\
\hline Chronic renal insufficiency, n (\%) & $21(23.1)$ \\
\hline \multicolumn{2}{|l|}{ BMI (kg/m²), n (\%) } \\
\hline$\leq 24.9$ & $31(34.4)$ \\
\hline$>24.9$ & $59(65.6)$ \\
\hline Oral inotropic agents, n (\%) & $16(17.8)$ \\
\hline Active smoking & $52(57.8)$ \\
\hline Q wave infarction history & $12(13.3)$ \\
\hline Statin therapy & $7 \mid(78.9)$ \\
\hline Previous cardiac surgery & $37(4 I .1)$ \\
\hline Hypercholesterolemia & $70(77.8)$ \\
\hline \multicolumn{2}{|l|}{ Preprocedure cardiac rhythm } \\
\hline SR & $65(72.2)$ \\
\hline AF & $21(23.3)$ \\
\hline Pacemaker & $4(4.4)$ \\
\hline Preprocedural coronary angiography & $79(87.8)$ \\
\hline \multicolumn{2}{|l|}{ LV ejection fraction } \\
\hline Good & $53(58.9)$ \\
\hline Moderate & $28(31.1)$ \\
\hline Poor & $9(10.0)$ \\
\hline \multicolumn{2}{|l|}{$\begin{array}{l}\text { Duration of postprocedural inotropic } \\
\text { support (hours) }\end{array}$} \\
\hline 0 & $72(81.8)$ \\
\hline$<12$ & $7(8.0)$ \\
\hline $12-24$ & $3(3.4)$ \\
\hline$>24$ & $6(6.8)$ \\
\hline
\end{tabular}

Abbreviations: AF, atrial fibrillation; $\mathrm{BMI}$, body mass index; IQR, interquartile range; LV, left ventricular; SR, sinus rhythm.
Table 2 Thirty-day incidence of cardiac complications post TAVI

\begin{tabular}{ll}
\hline Cardiac complications & $\mathbf{n}(\%)$ \\
\hline Overall & $17(18.9)$ \\
AV block requiring PPM implantation & $3(3.3)$ \\
Cardiac tamponade & $2(2.2)$ \\
NOAF & $10(11.1)$ \\
Pulseless VT and/or VF & $2(2.2)$ \\
\hline
\end{tabular}

Abbreviations: AV, atrioventricular; NOAF, new-onset atrial fibrillation; PPM, permanent pacemaker; TAVI, transcatheter aortic valve implantation; VF, ventricular fibrillation; VT, ventricular tachycardia.

shockable cardiac arrest rhythm (pulseless VT and/or VF) and the remaining $2(2.2 \%)$ developed cardiac tamponade.

Table 3 provides the main associations between patient characteristics and cardiac complications resulting from bivariate analyses. Bivariate analysis revealed that sinus and pacemaker preprocedural cardiac rhythm, post TAVI tracheal intubation and receiving of oral inotropic agents preprocedurally and intravenous inotropes postprocedurally were the main risk factors for cardiovascular complications after transapical TAVI. Specifically, patients who were intubated postprocedurally ( $36.4 \%$ vs $8.8 \%, p=0.001)$ and those receiving inotropic agents, either orally during the preprocedural phase $(43.8 \%$ vs $13.5 \%, p=0.01)$ or intravenously during the post TAVI period $(43.8 \%$ vs $13.9 \%, p=0.01$ ), are characterized by significantly greater probability for postprocedural cardiac morbidity. However, patients with preexisting AF had significantly lower incidence of cardiac complications compared with those on sinus or pacemaker rhythm ( $0 \%$ vs $24.6 \%, p=0.01)$.

\section{Discussion}

The main findings of our study were both the estimated (overall and complication type) incidence of cardiac complications following transapical TAVI procedure and the identification of four patient-related variables as the main predictors of cardiac complications among recipients of transapical TAVI. The need for tracheal intubation postprocedurally, the absence of preexisting AF, receiving of oral inotropes preprocedurally and the need for postprocedural intravenous inotropic support were significantly associated with the higher incidence of cardiac complications after TAVI.

Not surprisingly, parameters such as the prolonged postprocedural mechanical ventilatory and intravenous inotropic support, along with oral inotropic therapy, prior TAVI are risk factors for patient morbidity, probably reflecting a worse general postprocedural clinical status of these patients. Besides, research on TAVI patient series, has consistently found that patients who require mechanical ventilatory support, ${ }^{16,17}$ and 
Table 3 Bivariate analyses between independent variables and cardiac complications

\begin{tabular}{|c|c|c|c|}
\hline \multirow[t]{2}{*}{ Independent variables } & \multicolumn{2}{|c|}{ Cardiac complications n (\%) } & \multirow[t]{2}{*}{$P$-value } \\
\hline & No & Yes & \\
\hline Gender & & & $0.8^{\mathrm{a}}$ \\
\hline Male & $36(80.0)$ & $9(20.0)$ & \\
\hline Female & $37(82.2)$ & $8(17.8)$ & \\
\hline Age (years) & $80.8(7.8)$ & $83.9(5.2)$ & $0.13^{b}$ \\
\hline EuroSCORE (\%) & $21.8(12.7)$ & $28.4(14.6)$ & $0.07^{\mathrm{b}}$ \\
\hline $\mathrm{Q}$ wave infarction history & & & $0.3^{\mathrm{a}}$ \\
\hline Yes & II (9I.7) & I (8.3) & \\
\hline No & $62(79.5)$ & $16(20.5)$ & \\
\hline Previous cardiac surgery & & & $0 . I^{\mathrm{a}}$ \\
\hline Yes & $33(89.2)$ & $4(10.8)$ & \\
\hline No & $40(75.5)$ & $13(24.5)$ & \\
\hline Diabetes & & & $0.6^{\mathrm{a}}$ \\
\hline Yes & $17(85)$ & $3(15)$ & \\
\hline No & $56(80.0)$ & $14(20.0)$ & \\
\hline Hypercholesterolemia & & & $0.25^{\mathrm{a}}$ \\
\hline Yes & $55(78.6)$ & $15(21.4)$ & \\
\hline No & $18(90.0)$ & $2(10.0)$ & \\
\hline Statin therapy & & & $0.3^{\mathrm{a}}$ \\
\hline Yes & $56(78.9)$ & $15(2 \mid .1)$ & \\
\hline No & $17(89.5)$ & $2(10.5)$ & \\
\hline Active smoking & & & $0.6^{\mathrm{a}}$ \\
\hline Yes & $43(82.7)$ & $9(17.3)$ & \\
\hline No & $30(78.9)$ & $8(21.1)$ & \\
\hline BMI $\left(\mathrm{kg} / \mathrm{m}^{2}\right)$ & & & $0.6^{\mathrm{a}}$ \\
\hline$\leq 24.9$ & $26(83.9)$ & $5(16.1)$ & \\
\hline$>24.9$ & $47(79.7)$ & $12(20.3)$ & \\
\hline Creatinine levels (mg/dL) & & & $0.15^{\mathrm{a}}$ \\
\hline$\leq 1.4$ & $47(77.0)$ & $14(23.0)$ & \\
\hline$>1.4$ & $26(89.7)$ & $3(10.3)$ & \\
\hline Chronic renal insufficiency & & & $0.1^{c}$ \\
\hline Yes & $20(95.2)$ & $\mathrm{I}(4.8)$ & \\
\hline No & $53(76.8)$ & $16(23.2)$ & \\
\hline Preprocedural cardiac rhythm & & & $0.0 I^{c}$ \\
\hline SR and pacemaker & $52(75.4)$ & $17(24.6)$ & \\
\hline $\mathrm{AF}$ & $21(100.0)$ & $0(0.0)$ & \\
\hline Preprocedural coronary angiography & & & $1.0^{c}$ \\
\hline Yes & $64(81.0)$ & $15(19.0)$ & \\
\hline No & $9(81.8)$ & $2(18.2)$ & \\
\hline LV ejection fraction & & & $0.03^{d}$ \\
\hline Good & $45(84.9)$ & $8(15.1)$ & \\
\hline Moderate & $24(85.7)$ & $4(14.3)$ & \\
\hline Poor & $4(44.4)$ & $5(55.6)$ & \\
\hline Oral inotropic agents & & & $0.0 I^{c}$ \\
\hline No & $64(86.5)$ & $10(13.5)$ & \\
\hline Yes & $9(56.3)$ & $7(43.8)$ & \\
\hline Duration of postprocedural inotropic support (hours) & & & $0.0 I^{c}$ \\
\hline 0 & $62(86.1)$ & $10(13.9)$ & \\
\hline$>0$ & $9(56.3)$ & $7(43.8)$ & \\
\hline Postprocedural blood loss volume $(\mathrm{mL})$ & & & $0.03^{\mathrm{a}}$ \\
\hline 0 & $39(90.7)$ & $4(9.3)$ & \\
\hline$>0$ & $34(72.3)$ & $13(27.7)$ & \\
\hline Postprocedural intubation time (hours) & & & $0.00 I^{a}$ \\
\hline 0 & $52(9 \mid .2)$ & $5(8.8)$ & \\
\hline$>0$ & $21(63.6)$ & $12(36.4)$ & \\
\hline
\end{tabular}

Notes: Continuous variables are presented as mean (SD), while categorical variables are presented as $\mathrm{n}(\%) .{ }^{\mathrm{a}} \chi^{2}$ test; ${ }^{\mathrm{b}} \mathrm{Student}$ 's $t$-test; 'Fisher's exact test; ${ }^{\mathrm{d}} \chi^{2}$ trend test; Bold entries denote $p \leq 0.01$.

Abbreviations: AF, atrial fibrillation, BMI, body mass index, LV, left ventricular, SR, sinus rhythm. 
those at New York Heart Association (NYHA) class III/IV ${ }^{11}$ have poorer outcome, with higher morbidity and mortality rates.

Although intravenous inotropic support is suggested as a standard intervention for TAVI patients with low cardiac output and hemodynamic instability, mainly during the early postprocedural phase, ${ }^{18}$ literature has not consistently investigated its potential negative effects on patient outcome, in terms of higher incidence of cardiac complications. It seems that the arrhythmogenic adverse effects of inotropic agents could explain the higher incidence of cardiac complications, in terms of rhythm disturbances. ${ }^{18}$ However, in other patient series, such as cardiac surgical patients, postoperative support with intravenous inotropic agents is a well-known predictor of poor outcome, including significantly higher incidence of cardiac complications. ${ }^{19}$

However, we found that patients who had sinus or pacemaker rhythm preprocedurally are characterized by significantly greater probability for cardiac complications after TAVI, compared with those with preexisting AF (24.6\% vs $0 \%$ ). Indeed, no one of those with preexisting AF developed any cardiac complication in our patients' series. Other studies have shown that preexisting AF is a well-documented risk factor for poorer mid- and long-term clinical outcome among TAVI patients, even if patient outcome is similar between patients with preexisting AF and those with sinus rhythm during the first 30-day postprocedural period. ${ }^{12}$

The above-mentioned unexpected finding could be explained by our study methods. Specifically, we considered NOAF as the occurrence of AF in patients who had sinus rhythm preprocedurally, excluding apriori the patients with preexisting AF from this cardiac complication type. This limitation, in conjunction with the small sample size of the present study, could justify this seemingly paradox finding.

Postprocedural atrioventricular block requiring a PPM implantation is the main cardiac complication, which has been widely investigated in the currently published researches, ${ }^{6,10,20,21}$ with, contrary to our results, an incidence of $13.4 \%$ according to a recently published systematic review involving 11,599 patients. ${ }^{8}$ Previous published research has consistently found that procedure-related parameters such as the implantation of a larger prosthesis into a smaller annulus and a deep valve implantation, but also patient-related ones included a pre-existing right bundle block, patient age $>75$ years, male gender, chronic AF, perioperative bradycardia and high calcification load of the native valve are among the main risk factors for postprocedural heart block. ${ }^{7,22}$ The lower incidence of heart block with PPM implantation in our cohort could be explained by its small sample size that could affect the validity of the present study.

NOAF following TAVI procedures is a well-known complication, ${ }^{23}$ while transapical approach has been identified as an independent predictor of this patient outcome. ${ }^{12}$ It seems that the more invasive character of transapical TAVIs accompanied by epicardial and pericardial injury due to transapical access route could justify this association. ${ }^{12,24}$ Several studies have reported a NOAF incidence of $1 \%-32 \%$ postprocedurally, ${ }^{12,23,25,26}$ a finding that is consistent with the present study.

As aforementioned, another important cardiac complication in our cohort was the occurrence of malignant ventricular arrhythmias (pulseless VT and/or VF) during the post-TAVI period in 2 patients. Our incidence is similar to the relevant early outcomes of other recently published studies. ${ }^{27,28}$ Although the incidence of major ventricular arrhythmias after TAVI is lower than the pre-TAVI rates, ${ }^{27}$ post-TAVI conditions such as the aggressive left ventricular remodeling, ${ }^{28}$ the higher systolic ventricular overload, the reduced systolic function and the left ventricular hypertrophy ${ }^{27}$ could interpret the presentation of ventricular adverse events postprocedurally. In addition, the local tissue injury and edema due to transapical manipulations could trigger the above-mentioned disorders. ${ }^{29}$

According to the findings of our study, 2 patients (2.2\%) were developed cardiac tamponade postprocedurally, a major complication that has been associated with high mortality rates. ${ }^{30}$ Factors associated with the applied technique and devices, and patient-related conditions, such as calcified aortic annulus and fragile myocardium due to severe ischemic heart disease are the main predisposing factors for hemopericardium and cardiac tamponade after TAVI. ${ }^{30}$ Our incidence is in line with the currently available published researches. ${ }^{30,31}$ It is worth mentioning that other studies did not give a clear picture on cardiac tamponade incidence as complication post TAVI, considering hemopericardium, surgical re-exploration due to bleeding and cardiac tamponade as the same clinical condition. For instance, Čanádyová et al reported an incidence of $9.7 \%$ of surgical re-exploration due to bleeding or cardiac tamponade, without clarification on the size of these patient subgroups. ${ }^{32}$ In addition, Margolina et al in their study of 99 patients who underwent TAVI in a single center in Russia stated hemopericardium, with an incidence of $6 \%$, among the main complications after TAVI, without giving information whether this condition led to cardiac tamponade. ${ }^{33}$

\section{Study limitations}

The present study adds new data to the current knowledge regarding the periprocedural factors associated with 
increased probability for cardiac complications following TAVI, through the transapical approach. However, some limitations should be acknowledged. First, this single-center study, with a relatively small sample size and retrospective design, carries all the limitations adherent to such an investigation and could influence the internal and external validity of the study findings. Second, the small sample size of the present study did not allow us to use multivariate statistical models to analyze our data. Third, mid- and long-term data were not addressed, according to our study design. Finally, patients undergoing TAVI with other than transapical access route were not included in our study sample.

\section{Conclusion}

TAVI procedure is an effective and main-stream option for inoperable, high and intermediate surgical risk patients with severe, symptomatic aortic stenosis, characterized by an increasing number of recipients annually, since its introduction into clinical practice in $2002 .{ }^{8}$ Despite the documented effectiveness and safety of this procedure, TAVIs are accompanied by, among others, cardiac complications during the postprocedural period, which must be treated immediately and optimally, ensuring the best patient outcome.

Patients remained intubated and under intravenous inotropic support during the postprocedural phase, those with sinus or pacemaker rhythm at baseline and patients who receive oral inotropes preprocedurally have significantly higher probability to develop cardiac complications post TAVI. The early identification of these high-risk patient groups could allow clinicians to have a better patient care planning and to take measures rapidly aiming to manage severe and life-threatening complications. Furthermore, administrators, taking into account the above information, could achieve the optimal allocation of the limited health care system resources. Finally, based on the above-mentioned study limitations, further research, including multi-center, prospective studies, with greater sample size, mid- and long-term follow-up data and multivariate data analysis, is needed.

\section{Disclosure}

The authors report no conflicts of interest in this work.

\section{References}

1. Leon MB, Smith CR, Mack M, et al; PARTNER Trial Investigators. Transcatheter aortic-valve implantation for aortic stenosis in patients who cannot undergo surgery. NEngl J Med. 2010;363(17):1597-1607.

2. Smith CR, Leon MB, Mack MJ, et al. Transcatheter versus surgical aortic-valve replacement in high-risk patients. $N$ Engl J Med. 2011;364(23):2187-2198.
3. Leon MB, Smith CR, Mack MJ, et al. Transcatheter or surgical aortic-valve replacement in intermediate-risk patients. $N$ Engl J Med. 2016;374(17):1609-1620.

4. Reardon MJ, Van Mieghem NM, Popma JJ, et al. Surgical or transcatheter aortic-valve replacement in intermediate-risk patients. $N$ Engl J Med. 2017;376(14):1321-1331.

5. Holmes DR Jr, Nishimura RA, Grover FL, et al. Annual outcomes with transcatheter valve therapy: from the STS/ACC TVT registry. J Am Coll Cardiol. 2015;66(25):2813-2823.

6. D’Onofrio A, Salizzoni S, Filippini C, et al. Transapical aortic valve replacement is a safe option in patients with poor left ventricular ejection fraction: results from the Italian Transcatheter Balloon-Expandable Registry (ITER). Eur J Cardiothorac Surg. 2017;52(5):874-880.

7. Neragi-Miandoab S, Michler RE. A review of most relevant complications of transcatheter aortic valve implantation. ISRN Cardiol. 2013;2013:956252.

8. Chakos A, Wilson-Smith A, Arora S, et al. Long term outcomes of transcatheter aortic valve implantation (TAVI): a systematic review of 5-year survival and beyond. Ann Cardiothorac Surg. 2017;6(5):432-443.

9. Liao YB, Deng XX, Meng Y, et al. Predictors and outcome of acute kidney injury after transcatheter aortic valve implantation: a systematic review and meta-analysis. EuroIntervention. 2017;12(17):2067-2074.

10. Bajrangee A, Coughlan JJ, Teehan S, et al. Early and mid-term outcomes after transcatheter aortic valve implantation (TAVI) in Ireland. Int $J$ Cardiol Heart Vasc. 2017;16:1-3.

11. Gilard M, Schlüter M, Snow TM, et al. The 2011-2012 pilot European Society of Cardiology Sentinel Registry of Transcatheter Aortic Valve Implantation: 12-month clinical outcomes. EuroIntervention. 2016;12(1):79-87.

12. Sannino A, Gargiulo G, Schiattarella GG, et al. A meta-analysis of the impact of pre-existing and new-onset atrial fibrillation on clinical outcomes in patients undergoing transcatheter aortic valve implantation. EuroIntervention. 2016;12(8):e1047-e1056.

13. Xiong TY, Liao YB, Zhao ZG, et al. Causes of death following transcatheter aortic valve replacement: a systematic review and meta-analysis. J Am Heart Assoc. 2015;4(9):e002096.

14. Patris V, Giakoumidakis K, Argiriou M, et al. Predictors of length of stay and duration of tracheal intubation after transcatheter aortic valve implantation. J Thorac Dis. 2017;9(4):1012-1022.

15. Eltheni R, Giakoumidakis K, Brokalaki H, Galanis P, Nenekidis I, Fildissis G. Predictors of prolonged stay in the intensive care unit following cardiac surgery. ISRN Nurs. 2012;2012:691561.

16. Kaier K, Reinecke H, Naci H, et al. The impact of post-procedural complications on reimbursement, length of stay and mechanical ventilation among patients undergoing transcatheter aortic valve implantation in Germany. Eur J Health Econ. 2018;19(2):223-228.

17. Goebel N, Ahad S, Schaeufele T, et al. Transcatheter aortic valve implantation in patients at extremely high risk of perioperative mortality. J Heart Valve Dis. 2015;24(5):635-639.

18. Huffmyer J, Tashjian J, Raphael J, Jaeger JM. Management of the patient for transcatheter aortic valve implantation in the perioperative period. Semin Cardiothorac Vasc Anesth. 2012;16(1):25-40.

19. Yamazaki Y, Oba K, Matsui Y, Morimoto Y. Vasoactive-inotropic score as a predictor of morbidity and mortality in adults after cardiac surgery with cardiopulmonary bypass. J Anesth. 2018;32(2):167-173.

20. Schroeter T, Linke A, Haensig M, et al. Predictors of permanent pacemaker implantation after Medtronic CoreValve bioprosthesis implantation. Europace. 2012;14(12):1759-1763.

21. Roten L, Wenaweser P, Delacrétaz E, et al. Incidence and predictors of atrioventricular conduction impairment after transcatheter aortic valve implantation. Am J Cardiol. 2010;106(10):1473-1480.

22. Siontis GC, Jüni P, Pilgrim T, et al. Predictors of permanent pacemaker implantation in patients with severe aortic stenosis undergoing TAVR: a meta-analysis. J Am Coll Cardiol. 2014;64(2):129-140.

23. Vavuranakis M, Kolokathis AM, Vrachatis DA, et al. Atrial fibrillation during or after TAVI: incidence, implications and therapeutical considerations. Curr Pharm Des. 2016;22(13):1896-1903. 
24. Panchal HB, Ladia V, Amin P, et al. A meta-analysis of mortality and major adverse cardiovascular and cerebrovascular events in patients undergoing transfemoral versus transapical transcatheter aortic valve implantation using Edwards valve for severe aortic stenosis. Am J Cardiol. 2014;114(12):1882-1890.

25. Jørgensen TH, Thygesen JB, Thyregod HG, Svendsen JH, Søndergaard L. New-onset atrial fibrillation after surgical aortic valve replacement and transcatheter aortic valve implantation: a concise review. J Invasive Cardiol. 2015;27(1):41-47.

26. Yankelson L, Steinvil A, Gershovitz L, et al. Atrial fibrillation, stroke, and mortality rates after transcatheter aortic valve implantation. Am J Cardiol. 2014;114(12):1861-1866.

27. Tempio D, Pruiti GP, Conti S, et al. Ventricular arrhythmias in aortic valve stenosis before and after transcatheter aortic valve implantation. Europace. 2015;17(7):1136-1140.

28. Papavasileiou LP, Halapas A, Chrisocheris M, et al. Sudden death after transcatheter aortic valve implantation. Are bradyarrhythmias always the cause? J Atr Fibrillation. 2015;8(3):1108.
29. Beinart R, Danik S, Palacios I, et al. Ventricular tachycardia following trans-apical aortic valve replacement. Europace. 2012;14(3):450-452.

30. Rezq A, Basavarajaiah S, Latib A, et al. Incidence, management, and outcomes of cardiac tamponade during transcatheter aortic valve implantation: a single-center study. JACC Cardiovasc Interv. 2012;5(12):1264-1272.

31. Zhao QM, Lognone T, Ivascau C, et al. Procedural results and 30-day clinical events analysis following Edwards transcatheter aortic valve implantation in 48 consecutive patients: initial experience. Chin Med $J$ (Engl). 2012;125(16):2807-2810.

32. Čanádyová J, Mokráček A, Pešl L, Kurfirst V, Šulda M. Short-term and medium-term outcomes of transapical aortic valve implantation as a single-strategy approach: one center's experience. Kardiochir Torakochirurgia Pol. 2015;12(2):95-102.

33. Margolina AA, Gruzdev KA, Lepilin MG, Tabakyan EA, Imaev TE, Akchurin RS. Complications after transcatheter aortic valve implantation. Kardiologiia. 2016;56(2):35-39.
Pragmatic and Observational Research

\section{Publish your work in this journal}

Pragmatic and Observational Research is an international, peer-reviewed open access journal that publishes data from studies designed to reflect more closely medical interventions in real-world clinical practice compared with classical randomized controlled trials (RCTs). The manuscript management system is completely online and includes a very quick and fair peer-review
Dovepress

system. Visit http://www.dovepress.com/testimonials.php to read real quotes from published authors.

Submit your manuscript here: https:/www.dovepress.com/pragmatic-and-observational-research-journal 